\title{
Article \\ Thermography as a Method to Detect Dental Anxiety in Oral Surgery
}

\author{
Roberta Gasparro ${ }^{1}$, Grazia Leonetti ${ }^{1}$, Michele Riccio ${ }^{2}\left(\right.$ ) , Andrea Irace ${ }^{2}\left({ }^{\circ}\right.$, Gilberto Sammartino ${ }^{1, *(1)}$, \\ Andrea Blasi ${ }^{1}$, Cristiano Scandurra ${ }^{1} \mathbb{C}$, Nelson Mauro Maldonato ${ }^{1}{ }^{\mathbb{C}}$, Pasquale Sammartino ${ }^{1}$ \\ and Gaetano Marenzi ${ }^{1}$ \\ 1 Department of Neuroscience, Reproductive Science and Dentistry, University of Naples Federico II, \\ 80131 Naples, Italy; roberta.gasparro@unina.it (R.G.); gr.leonetti@studenti.unina.it (G.L.); \\ andrea.blasi@unina.it (A.B.); cristiano.scandurra@unina.it (C.S.); nelsonmauro.maldonato@unina.it (N.M.M.); \\ pasqualesammartino91@gmail.com (P.S.); gaetano.marenzi@unina.it (G.M.) \\ 2 Department of Electrical Engineering and Information Technology, University of Naples Federico II, \\ 80125 Naples, Italy; michele.riccio@unina.it (M.R.); andrea.irace@unina.it (A.I.) \\ * Correspondence: gilberto.sammartino@unina.it; Tel.: +39-081-746-2118
}

Citation: Gasparro, R.; Leonetti, G.; Riccio, M.; Irace, A.; Sammartino, G.; Blasi, A.; Scandurra, C.; Maldonato, N.M.; Sammartino, P.; Marenzi, G. Thermography as a Method to Detect Dental Anxiety in Oral Surgery. Appl. Sci. 2021, 11, 5421. https://doi.org/ 10.3390/app11125421

Academic Editor: Bruno Chrcanovic

Received: 24 May 2021

Accepted: 8 June 2021

Published: 10 June 2021

Publisher's Note: MDPI stays neutral with regard to jurisdictional claims in published maps and institutional affiliations.

Copyright: (c) 2021 by the authors. Licensee MDPI, Basel, Switzerland. This article is an open access article distributed under the terms and conditions of the Creative Commons Attribution (CC BY) license (https:// creativecommons.org/licenses/by/ $4.0 /)$.

\begin{abstract}
Background: the aim of this study was to evaluate if dental anxiety can be measured objectively using thermal infrared imaging. (2) Methods: Patients referred to the Department of Oral Surgery of the University of Naples Federico II and requiring dental extractions were consecutively enrolled in the study. Face thermal distribution images of the patients were acquired before and during their first clinical examination using infrared thermal cameras. The data were analyzed in relation to five regions of interest (ROI) of the patient's face (nose, ear, forehead, zygoma, chin). The differences in the temperatures assessed between the two measurements for each ROI were evaluated by using paired T-test. The Pearson correlation and linear regression were performed to evaluate the association between differences in temperatures and Modified Dental Anxiety Scale (MDAS) questionnaire score, age, and gender; (3) results: sixty participants were enrolled in the study (28 males and 32 females; mean age 57.4 year-old; age range 18-80 year-old). Only for nose and ear zone there was a statistically significant difference between measurements at baseline and visit. Correlation between the thermal imaging measurements and the scores of the MDAS questionnaire was found for nose and ear, but not for all of the other regions. (4) Conclusions: the study demonstrated a potential use of thermal infrared imaging to measure dental anxiety.
\end{abstract}

Keywords: dental anxiety; thermal imaging; thermal cameras; facial temperature

\section{Introduction}

Dental anxiety can be considered as an aversive emotional state of worry or apprehension in anticipation of dental treatment [1,2]. It has been cited as the fifth most common cause of anxiety by Agras et al. [3]. For Beaton et al. [4] dental anxiety, or dental fear, is estimated to affect approximately $36 \%$ of the population, with a further $12 \%$ suffering from extreme dental fear. This anxiety can have serious repercussions in terms of oral health and quality of life and could be a significant barrier to dental attendance [5]. Thus, identifying during the preoperative phase patients with high levels of dental anxiety is of great benefit to both clinicians and patients. Indeed, the underestimation of a patient's state of apprehension can affect the correct and/or complete execution of the treatment with consequent dissatisfaction on both patients and dentists.

The identification of dental anxiety can be realized by means of subjective and objective methods. Subjective measurements are based on multiple and single item self-reporting questionnaires, such as Corah's Dental Anxiety Scale (CDAS) [6], the Modified Dental Anxiety Scale (MDAS) [7] or Kleinknecht Dental Fear Survey (DFS) [8]. They are the most commonly used questionnaires and have been shown to be reliable and valid in 
multiple languages. The advantages of these methods are the disclosure of the degree of anxiety (mildly anxious, moderately anxious, extremely anxious or dental-phobic) but their routine use is very limited because of the lack of time and expertise in their interpretation. Furthermore, self-report questionnaires suffer from a significant bias: they request respondents to be aware of their own anxious states, as well as to be free from social desirability. Therefore, it may happen that the levels of anxiety reported in the answers are not actually those experienced by patients. Objective measurements, instead, involve the assessment of blood pressure, pulse rate, or galvanic skin response [9-11]. The objective methods are more accurate in the measurement of dental anxiety and they confirm the presence of physiological changes in the body as the result of the stress and anxiety caused by dental procedures. However, even these methods involve some disadvantages: the pulse rate, for example, may be altered as a result of the physical movements of the patient during dental treatment and the galvanic skin response may provide too high a level of sensitivity, giving false positive results. Furthermore, all these methods require a direct contact with the patient's body, which may cause discomfort and distress, so increasing the level of anxiety [9].

Therefore, we have proposed thermal imaging as a new technique for the identification of dental anxiety, a contactless imaging technique widely known in medicine and successfully used in the diagnosis of breast cancer [12], diabetic neuropathy [13] and peripheral vascular disorders [14]. In fact, abnormalities such as malignancies, inflammation, infection and anxiety cause localized increases in temperature shown at thermal imaging as hot spots or as asymmetrical patterns in an infrared thermogram [15]. Even though it is non-specific, infrared thermography is a powerful tool for the detection of problems that affect a patient's physiology [16]. In dentistry, thermography has been used to assess subjects with chronic orofacial or temporomandibular joint pain, or inferior alveolar nerve deficits or to monitor changes in bone temperature during the drilling process [17-19]. To the best of our knowledge, this is the first application of thermal infrared imaging as a method for the identification of dental anxiety in oral surgery. The aim of this study was to ascertain whether facial thermograms can be used as a valid and reliable somatic indicator of dental anxiety.

\section{Materials and Methods}

\subsection{Study Design}

The study was designed as a clinical validation model of thermography in the detection of dental anxiety in comparison with the MDAS questionnaire (Figure 1). Changes in facial skin temperature were expressed as differences between the initial and final temperature. The results of the MDAS questionnaire were compared with the facial temperature changes.

\subsection{Participants}

Between September 2019 and December 2019, patients who had accessed the Oral Surgery Unit of the University of Naples Federico II for the day hospital, ordinary hospitalization or pre-recovery were invited to take part in the study during their first appointment. The inclusion criteria were: (1) age between 18 and 80 years; (2) ability to understand and sign the informed consent; and (3) first appointment at the Department of Dental Surgery to undergo dental surgery (4) patients needed the extraction of fully erupted tooth. The exclusion criteria were: (1) age under 18 or over 80 years, (2) inability to understand and sign the informed consent, (3) a diagnosed psychiatric pathology (assessed by asking the clinical history), (4) abuse of alcohol or drugs or (5) pregnancy or lactation. The study protocol was approved by the Ethical Committee of the University of Naples Federico II (Protocol Number 187/19). The patients were carefully informed about the study and provided their written informed consent. The study was conducted according to the Principles of the Declaration of Helsinki on experimentation involving human subjects. 


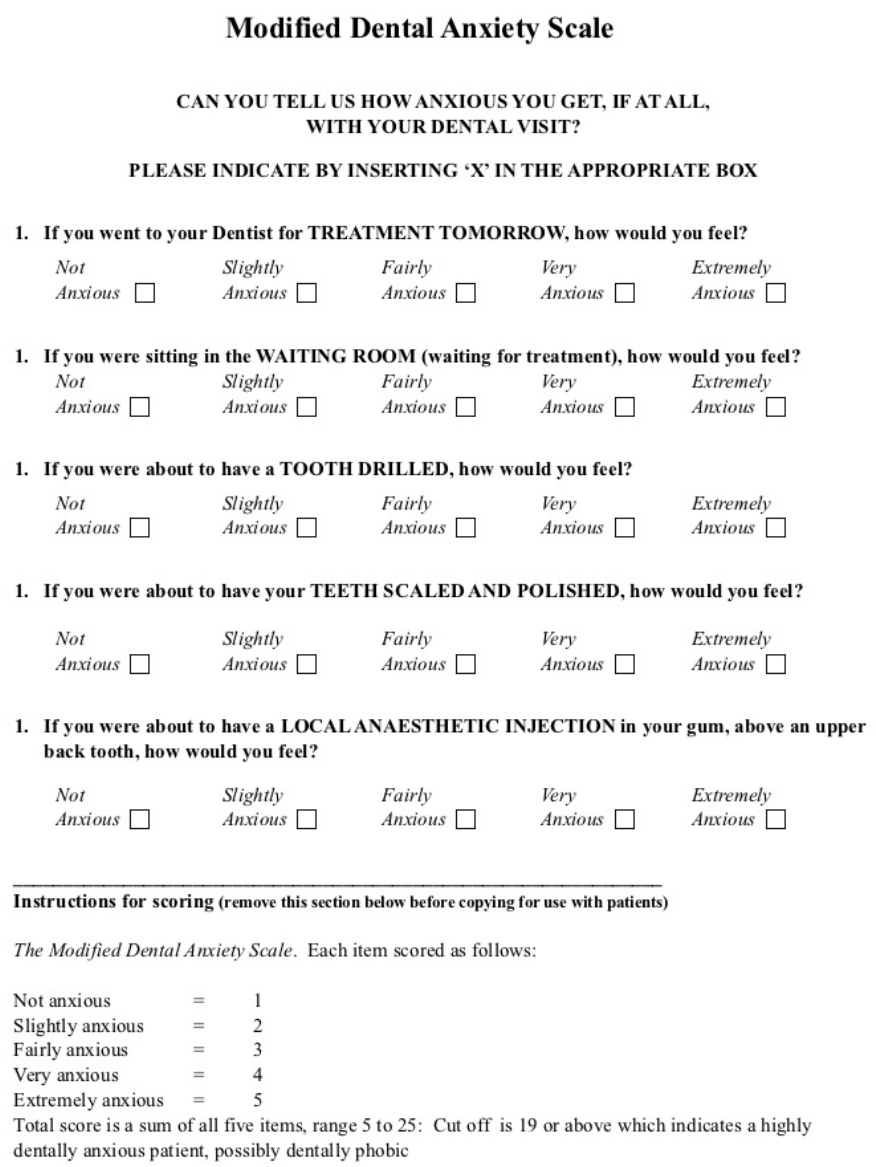

Figure 1. Modified Dental Anxiety Scale (MDAS) questionnaire (English version).

\subsection{Equipment}

For this study the platform InfraSensing by ServersCheck was selected. The configuration employed consisted of two thermal imaging sensors (ENV-THIMG-M, Zaventem, Belgium) connected to a base unit (SensorGateway, Zaventem, Belgium) acting as a network gateway. The thermal images were streamed to a personal computer and collected with a Matlab ${ }^{\circledR}$ graphical user interface developed at the Department of Electrical Engineering and Information Technology of Federico II University. The thermal imaging sensor: The selected temperature sensor is equipped with a focal plane array (FPA) consisting of $80 \times 60$ pixels active in the range $0-120{ }^{\circ} \mathrm{C}$ produced by the FLIR system (the LEPTON 2.5 module). The pixel size is $17 \mu \mathrm{m}$, while the thermal sensitivity is less than $50 \mathrm{mK}$. The thermal camera focuses on the temperature of individual items within its field of view ( $\mathrm{FoV}$ ) of $50^{\circ}$ at a maximum distance of $45 \mathrm{~m}$. Each sensor is connected to the base unit using a standard network cable. The network cable transmits data and power between the base unit and the sensor. The recording frame rate was $1.5 \mathrm{~s}$.

The base unit-sensor gateway: The base unit has a built-in web server. This enables the operator to perform several activities including configuring reading sensor data and defining alerts.

The MATLAB interface: The data stream between each thermal imaging sensor and the PC is regulated by an ad-hoc Matlab software. The interface allows you to send controls from the PC in order to acquire and elaborate thermal images. It is possible to save these images in both picture and video formats. Moreover, the interface is provided with some edit control keys used to set-up the interface, such as the Camera ID, IP address or acquisition period and frame-rate. In addition, it provides the temperatures of a selected area of the thermal image (a ROI) and allows different kinds of post-process elaboration (Figure 2). 


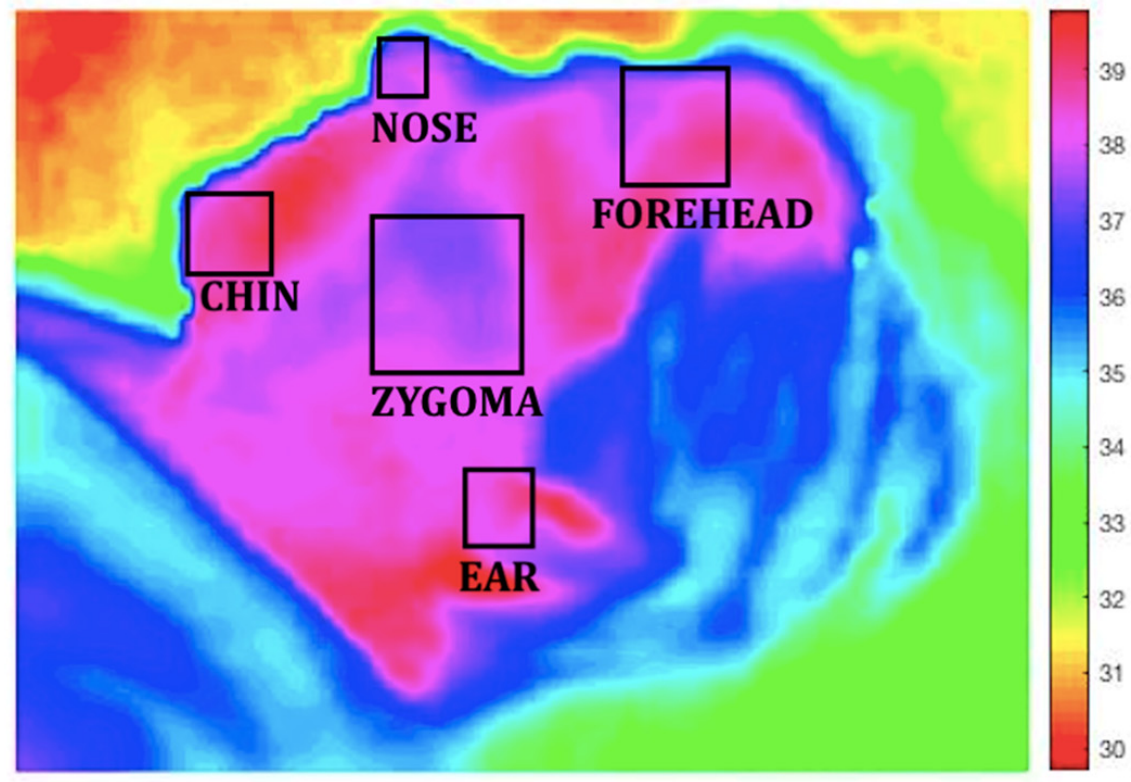

Figure 2. Regions of interest (ROIs).

\subsection{Procedures}

The thermal recordings were performed in a controlled environment: the temperature was maintained in a range from 18 to $25^{\circ} \mathrm{C}$ and the humidity was controlled. The door room was closed, the room was sound-proofed and no one was allowed to enter during the thermal registration except for the staff selected. All topical applications such as ointments and cosmetics and all objects like jewelry and glasses were removed from all the relevant areas of the face. The room was free from any secondary infrared sources such as an incandescent lamp or direct sun-light and the cameras were placed perpendicular to patients' face at a distance of $30 \mathrm{~cm}$. The thermal recording of the patient was divided into two steps. The first step called the BASELINE was used to acquire a starting temperature. During this step the patient sat on the dentist's chair and no stimuli were given. Only the patient and the thermal camera operator were present in the room. This recording started as soon as the patient entered the room and ended two minutes later. The second step was called the VISIT. This thermal recording started as soon as the dentist entered the room and continued during the anamnestic and clinical examination and the explanation of the treatment plan. For all the patients, a single dentist, unaware of the aim of the study, performed the examinations. On the face of each subject five ROIs centered on the nose, ear, cheek, forehead, and chin were taken into consideration. A temperature was recorded for each ROI. At the end of the thermal recordings a difference of temperature for each ROI between the temperature recorded during the BASELINE step and the temperature recorded during the VISIT step was calculated and averaged, respectively. After the medical examination the patient filled out the Italian version of the MDAS questionnaire 20 to assess the presence and severity of dental anxiety. This is a five-item scale measuring dental anxiety on a five-point Likert scale and generating a total score ranging from 5 (not anxious) to 25 (extremely anxious). The cut-off of 19 points may identify patients with high dental anxiety.

In order to classify the patients, the following criteria were considered: (1) a difference of temperature between $0.1^{\circ} \mathrm{C}$ and $0.3^{\circ} \mathrm{C}$, relating to an average difference of temperature of $0.2{ }^{\circ} \mathrm{C}$, identified a non-significant difference of temperature attributable to patients with no dental anxiety; (2) a difference of temperature between $0.3^{\circ} \mathrm{C}$ and $0.9{ }^{\circ} \mathrm{C}$, relating to an average difference of temperature of $0.6{ }^{\circ} \mathrm{C}$, identified a significant difference of temperature attributable to patients with a medium dental anxiety; and (3) a difference of temperature between $1^{\circ} \mathrm{C}$ and $2^{\circ} \mathrm{C}$, relating to an average difference of temperature of 
$1.5^{\circ} \mathrm{C}$, identified a significant difference of temperature attributable to patients with high dental anxiety.

For the MDAS score the following criteria were used: (1) a score from 5 to 10 points identified patients with no dental anxiety or with a mild dental anxiety, categorized as non-significant dental anxiety (Group 1); (2) a score from 11 to 18 points identified patients affected by moderate dental anxiety (Group 2); and (3) a score from 20 to 25 points identified patients affected by severe dental anxiety (Group 3).

\subsection{Data Analysis}

The measurements of temperature at the baseline step and the visit step were considered for the final analysis. The differences in the temperatures assessed between the two measurements for each ROI were evaluated by using paired $t$-test. The Pearson correlation was performed to evaluate the association between differences in temperatures and MDAS questionnaire score, age, and gender for each ROI. The three MDAS groups were used (i.e., non-significant, moderate, and severe dental anxiety). Multivariate linear regression was conducted to evaluate the association between temperature changes and age, gender, and three MDAS groups. A commercially available statistical software (IBM SPSS v.22.0, IBM, Armonk, NY, USA) was used to conduct all the statistical analyses in this study, and $p$-value $<0.05$ (two-tails) was considered as statistically significant.

\section{Results}

Sixty participants were enrolled in the study. Twenty-eight males and thirty-two females were recruited, and the mean age was 57.4 years-old (ranging from 18 to 80 yearsold). Furthermore, $46(76.7 \%)$ patients had an educational level $\leq$ high school, while $14(23.3 \%) \geq$ university college. All the patients correctly completed all the steps of the study (Table 1).

Table 1. Characteristics of patient population.

\begin{tabular}{lccl}
\hline & Age (Mean \pm Std. Dev.) & Gender $(\mathbf{M} / \mathbf{F} \boldsymbol{n} ; \mathbf{\%})$ & $\begin{array}{l}\text { Education Level } \\
\text { (Degree Y/N; \%) }\end{array}$ \\
\hline Subjects $(\boldsymbol{n}=\mathbf{6 0})$ & $57.4 \pm 17.3$ & $28 / 32 ; 46.7 / 53.3$ & $46 / 14 ; 76.7 / 23.3$ \\
\hline Y: yes; N: no; std. dev: standard deviation; M: male; F: female.
\end{tabular}

Y: yes; N: no; std. dev: standard deviation; M: male; F: female.

Thirty-one participants had an MDAS score from 5 to 10 points (Group 1; nonsignificant dental anxiety), 14 participants from 11 to 18 points (Group 2; moderate dental anxiety), and 15 participants from 19 to 25 points (Group 3; severe dental anxiety). Only for nose and ear zone there was a statistically significant difference between measurements at baseline and visit $(p<0.001)$. No differences were found between two measurements at zygoma, forehead and chin region ( $p=0.109, p=0.182 ; p=0.347$ respectively) (Table 2). For nose region there was a statistical correlation between differences in temperature and MDAS scores $(p=0.001)$ but not for age $(p<0.001)$ and gender $(p=0.183)$. For ear region there was a statistical correlation between differences in temperature and MDAS scores $(p<0.001)$ and also for age $(p=0.032)$ but not for gender $(p=0.707)$ (Figures 3 and 4$)$. In all of the other regions, no significant correlations between temperatures, MDAS scores, age, and gender were found. The linear regression identified age as a significant predictor for temperature changes in ROI 2 (Beta $=-0.24, p=0.046$; R2 $=0.283$ ), and MDAS as a significant predictor for temperature changes in ROI 4 (Beta $=1.276, p=0.017 ; \mathrm{R} 2=0.168$ ) (Table 3). 
Table 2. Differences of temperature between baseline and visit for each ROI.

\begin{tabular}{cccccc}
\hline & $\begin{array}{c}\text { Roi 1 } \\
\text { (Nose) }\end{array}$ & $\begin{array}{c}\text { Roi 2 } \\
\text { (Ear) }\end{array}$ & $\begin{array}{c}\text { Roi 3 } \\
\text { (Zygoma) }\end{array}$ & $\begin{array}{c}\text { Roi 4 } \\
\text { (Forehead) }\end{array}$ & $\begin{array}{c}\text { Roi 5 } \\
\text { (Chin) }\end{array}$ \\
\hline $\begin{array}{c}\text { Temperature at baseline } \\
\text { (mean } \pm \text { std. dev.) }\end{array}$ & $33.3 \pm 2.7$ & $33.4 \pm 3.7$ & $34.6 \pm 2.8$ & $34.6 \pm 2.8$ & $34.8 \pm 2.8$ \\
$\begin{array}{c}\text { Temperature at visit } \mathbf{1} \\
\text { (mean } \pm \text { std. dev.) }\end{array}$ & $32.8 \pm 2.7$ & $33.1 \pm 2.6$ & $34.4 \pm 2.6$ & $34.4 \pm 2.5$ & $34.7 \pm 2.6$ \\
$\begin{array}{c}\text { Temperature changes } \\
\text { (mean } \pm \text { std. dev.) }\end{array}$ & $0.45 \pm 0.87$ & $0.25 \pm 1.03$ & $0.18 \pm 0.87$ & $0.15 \pm 0.86$ & $0.10 \pm 0.82$ \\
$\quad$ Significance $(\boldsymbol{p})$ & $<\mathbf{0 . 0 0 1}$ & $<\mathbf{0 . 0 0 1}$ & 0.109 & 0.183 & 0.347 \\
\hline std. dev: standard deviation; ROI: region of interest. & & &
\end{tabular}

std. dev: standard deviation; ROI: region of interest.
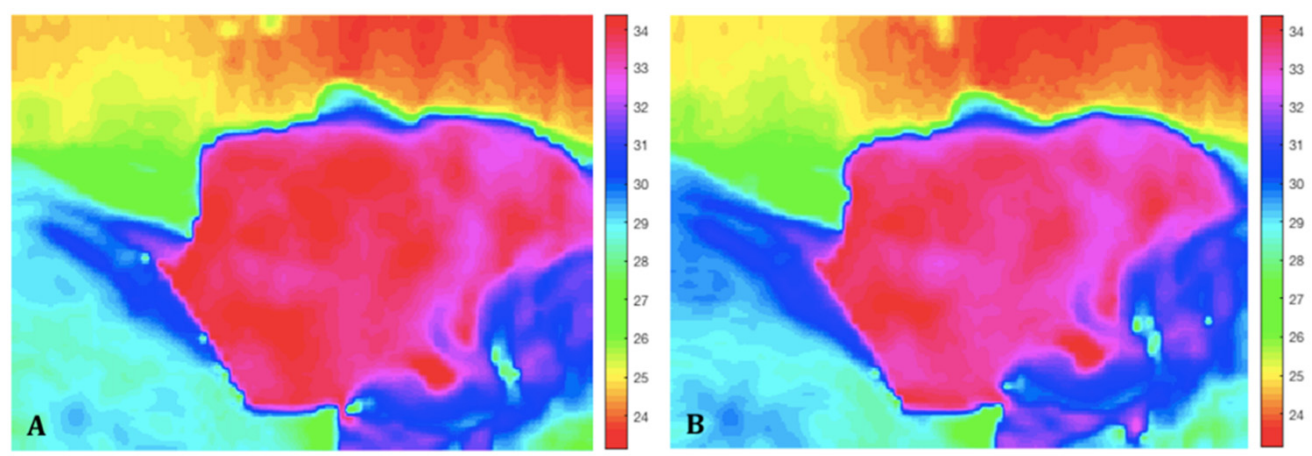

Figure 3. Baseline (A) and visit (B) thermal images in a patient with low dental anxiety. The thermal images show a lower change in temperature, that correspond to a patient with low score in the MDAS questionnaire.
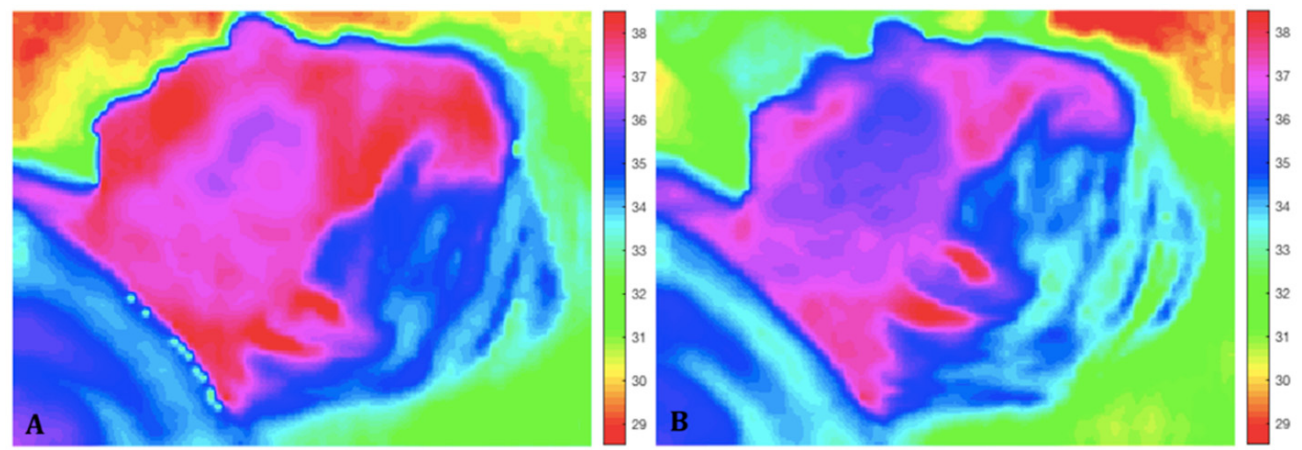

Figure 4. Baseline (A) and visit (B) thermal images in a patient with high dental anxiety. The colors between the two thermal recordings change in a significant way that correspond to a patient with a high score in the MDAS questionnaire.

Table 3. Correlation between differences of temperature, MDAS, age and gender.

\begin{tabular}{|c|c|c|c|c|c|c|}
\hline \multirow{2}{*}{ ROI } & \multirow{2}{*}{ Model } & \multicolumn{2}{|c|}{ Unstandardized Coefficients } & \multirow{2}{*}{$\begin{array}{l}\text { Standardized } \\
\text { Coefficients } \\
\text { Beta }\end{array}$} & \multirow{2}{*}{$t$} & \multirow{2}{*}{ Sig. } \\
\hline & & B & Standard Error & & & \\
\hline \multirow{6}{*}{ nose } & (Constant) & 0.221 & 0.583 & - & 0.378 & 0.707 \\
\hline & MDAS & -0.010 & 0.056 & -0.083 & -0.175 & 0.862 \\
\hline & Age & -0.012 & 0.006 & -0.240 & -2.046 & 0.046 \\
\hline & Gender & 0.215 & 0.198 & 0.129 & 1.083 & 0.284 \\
\hline & MDAS_LVL & 0.238 & 0.464 & 0.248 & 0.514 & 0.610 \\
\hline & MDAS_YN & 0.479 & 0.493 & 0.264 & 0.971 & 0.336 \\
\hline \multirow{6}{*}{ ear } & (Constant) & 0.574 & 0.596 & - & 0.963 & 0.340 \\
\hline & MDAS & -0.035 & 0.057 & -0.291 & -0.619 & 0.539 \\
\hline & Age & -0.013 & 0.006 & -0.261 & -2.241 & 0.029 \\
\hline & Gender & 0.002 & 0.203 & 0.001 & 0.008 & 0.994 \\
\hline & MDAS_LVL & 0.458 & 0.474 & 0.464 & 0.968 & 0.337 \\
\hline & MDAS_YN & 0.528 & 0.503 & 0.283 & 1.050 & 0.298 \\
\hline
\end{tabular}


Table 3. Cont.

\begin{tabular}{ccccccc}
\hline \multirow{2}{*}{ ROI } & Model & \multicolumn{2}{c}{ Unstandardized Coefficients } & $\begin{array}{c}\text { Standardized } \\
\text { Coefficients } \\
\text { Beta }\end{array}$ & $t$ & Sig. \\
& B & Standard Error & & & \\
\hline \multirow{5}{*}{ zygoma } & (Constant) & -0.005 & 0.608 & - & -0.008 & 0.994 \\
& MDAS & -0.092 & 0.058 & -0.819 & -1.576 & 0.121 \\
& Age & -0.009 & 0.006 & -0.190 & -1.479 & 0.145 \\
& Gender & 0.269 & 0.207 & 0.169 & 1.301 & 0.199 \\
& MDAS_LVL & 0.754 & 0.483 & 0.824 & 1.560 & 0.125 \\
& MDAS_YN & 0.192 & 0.514 & 0.111 & 0.374 & 0.710 \\
\hline \multirow{6}{*}{ forehead } & (Constant) & -0.005 & 0.608 & - & -0.008 & 0.994 \\
& MDAS & -0.092 & 0.058 & -0.819 & -1.576 & 0.121 \\
& Age & -0.009 & 0.006 & -0.190 & -1.479 & 0.145 \\
& Gender & 0.269 & 0.207 & 0.169 & 1.301 & 0.199 \\
& MDAS_LVL & 0.754 & 0.483 & 0.824 & 1.560 & 0.125 \\
& MDAS_YN & 0.192 & 0.514 & 0.111 & 0.374 & 0.710 \\
\hline \multirow{6}{*}{ chin } & (Constant) & -0.005 & 0.608 & - & -0.008 & 0.994 \\
& MDAS & -0.092 & 0.058 & -0.819 & -1.576 & 0.121 \\
& Age & -0.009 & 0.006 & -0.190 & -1.479 & 0.145 \\
& Gender & 0.269 & 0.207 & 0.169 & 1.301 & 0.199 \\
& MDAS_LVL & 0.754 & 0.483 & 0.824 & 1.560 & 0.125 \\
& MDAS_YN & 0.192 & 0.514 & 0.111 & 0.374 & 0.710 \\
\hline
\end{tabular}

\section{Discussion}

The aim of the current study has been to assess if thermography is a valid method to detect dental anxiety, using the MDAS questionnaire as a control. Although none of the anxiety questionnaires currently in use has been individuated as a gold standard, the MDAS is a brief, widely used, and well-validated instrument. Moreover, patients commonly report masking their anxiety, and therefore, evaluation with a brief anxiety questionnaire is considered beneficial and recommended, as it not only discloses the degree of anxiety but also appears to reduce it [20].

The findings of this study show that for nose and ear region there was a statistical correlation between differences in temperature and MDAS scores. Although the only significance correlation was found in these regions, other ROIs should be further investigated because probably in a larger sample they would be of interest and provide relevant information.

The aetiology of dental anxiety involves a variety of genetic, behavioural, and cognitive factors [21]. For instance, previous studies have found associations between high levels of dental anxiety and traumatic dental events (e.g., invasive treatments or excessive pain) [22-24]. However, another line of research has stressed the role that cognitive factors (e.g., vulnerability schema, patient's personal perceptions of the dentist and dental treatment, perceived lack of control) may have in the development of dental anxiety, independently from the past traumatic events [1,25-28]. Thus, these studies have demonstrated that dental anxiety, like other forms of anxiety, is a response to a danger or threat that activates the "fight or flight response", stimulating the organism to prepare for immediate action or self-protection [29]. When some sort of danger is perceived or anticipated, there is an activation of the autonomic nervous system and a relaxation of chemical hormones like adrenaline, noradrenaline and cortisol from the adrenal glands in the kidneys, which, in turn, provoke cardiovascular effects (increase in the heart rate and blood flow), respiratory effects (increase in the speed and depth of breathing), muscular effects and mental/behavioral effects [30-32]. Our study seems to demonstrate that changes in the blood flow and variations of the cutaneous temperature can be detected by thermal infrared imaging, that can record the intensity of radiation in the infrared part of the electromagnetic spectrum, converting it into a visible image, with the result that every pixel in the image is a temperature measurement [33].

The last five decades have witnessed a steady increase in the use of thermal imaging cameras to obtain correlations between the thermal physiology and skin temperature. 
Variations in the body temperature are a natural indicator of illness. Abnormalities such as malignancies, inflammation and infection cause localized increases in temperature which show as hot spots or as asymmetrical patterns in an infrared thermogram. Accordingly, this technique has been successfully used in the diagnosis of breast cancer, diabetes neuropathy and peripheral vascular disorders and in the detection of problems associated with gynaecology, kidney transplantation, dermatology, cardiology, neonatal physiology and fever screening [34].

Since 1867, when scientists first noticed that emotional excitement, such as a fit of anger, caused a general rise of temperature, thermography has been used to study psychological processes [35]. Initial thermographic research concentrated on the mental workload. Genno et al. conducted experiments in which the temperature of the skin was shown to have great potential as a physiological measure, because, when using a thermographic camera, no physical contact with the subject is needed [36]. In all patients, especially those with severe anxiety, the fight-or-flight response can induce medical emergencies, such as vasovagal syncope, hypertension or respiratory distress. In patients at risk, associated with pain, it can destabilize pre-existent pathologies [37].

Therefore, an early identification and subsequent management of dental anxiety is of great beneficial for both dentists and patients. Indeed, dentists who can early identify patients with high levels of dental anxiety could intervene effectively to reduce anxiety levels by creating a more containing setting for these patients or implementing a stronger shared decision-making process by agreeing on an appropriate treatment plan [38,39]. For example, dentists may encourage these patients to ask questions about the treatment and reassure them, or may help their patients in feeling to be in control over the situation, e.g., by agreeing on a signal (such as a raised hand) to interrupt the procedure in case of excessive anxiety. Creating optimal psychological and medical conditions, indeed, may reduce sources of stress, increasing patients' compliance in surgical interventions and post-operative care and, thus, increasing health outcomes. This seems particularly true in oral surgery, since invasive dental procedures (e.g., extractions or injections) are among the distressing dental events most associated with high dental anxiety [25].

However, the findings of the current study should be read in light of some limitations. First, the sample is not representative and was recruited from a single social reality, thus preventing the possibility to generalize our results. Secondly, we have assessed the validity of the method only by comparing it with the most frequently used questionnaire. It is possible that different results would have been obtained by using other objective and self-report measures.

Despite these limitations, the present study might have significant implications for future research and for clinic. Future developments could include the identification of dental anxiety during a dental procedure in relation to pain $[40,41]$, the understanding of the specific causes of stress, the monitoring of the increase in anxiety in "special needs" patients, the comparison of dental procedures in terms of stress and discomfort for the patient and the evaluation of the anxiety of the dentists themselves during the dental procedure. For example, future efforts may be focused on the development of cameras with an "alert signal" when a high level of anxiety raises during the procedures, indicating a possibility to interrupt the intervention, relaxing the patient and then continue. Despite the fact that this is a pilot study, the results are encouraging so, we could expect a confirmation of the method validation from more patients recruiting, but this is only an assumption. However, other studies are needed to confirm these findings.

\section{Conclusions}

Thermal imaging has a good capability in the objective identification and quantification of the presence of dental anxiety. Therefore, it can be considered as an innovative approach to the early detection and management of dental anxiety.

Author Contributions: Conceptualization, R.G. and G.M.; methodology, G.S.; software, A.B.; validation, A.I., G.S. and N.M.M.; formal analysis, A.B.; investigation, G.L.; resources, M.R. and A.I.; 
data curation, C.S. and A.B.; writing-original draft preparation, P.S. and G.L.; writing-review and editing, R.G. and C.S.; visualization, G.M.; supervision, G.S., A.I. and N.M.M.; project administration, G.S.; funding acquisition, M.R. and A.I. All authors have read and agreed to the published version of the manuscript.

Funding: This study was partially supported by a grant from MIUR (Dipartimenti di Eccellenza DM 11 May 2017 n.262) to the Department of Electrical Engineering and Information Technology, University of Naples Federico II.

Institutional Review Board Statement: The study protocol was approved by the Ethical Committee of the University of Naples Federico II (Protocol Number 187/19-date of approval 17 July 2019). The patients were carefully informed about the study and provided their written informed consent. The study was conducted according to the Principles of the Declaration of Helsinki on experimentation involving human subjects.

Informed Consent Statement: Informed consent was obtained from all subjects involved in the study.

Data Availability Statement: The data presented in this study are available on request from the corresponding author. The data are not publicly available due to ethical restrictions.

Conflicts of Interest: The authors declare no conflict of interest.

\section{References}

1. Armfield, J.M. Towards a better understanding of dental anxiety and fear: Cognitions vs. experiences. Eur. J. Oral. Sci. 2010, 118, 259-264. [CrossRef]

2. Facco, E.; Zanette, G. The Odyssey of Dental Anxiety: From Prehistory to the Present. A Narrative Review. Front. Psychol. 2017, 8, 1155. [CrossRef] [PubMed]

3. Agras, S.; Sylvester, D.; Oliveau, D. The epidemiology of common fears and phobia. Compr. Psychiatry 1969, 10, 151-156. [CrossRef]

4. Beaton, L.; Freeman, R.; Humphris, G. Why are people afraid of the dentist? Observations and explanations. Med. Princ. Pract. 2014, 23, 295-301. [CrossRef] [PubMed]

5. Hill, K.B.; Chadwick, B.; Freeman, R.; O'Sullivan, I.; Murray, J.J. Adult Dental Health Survey 2009: Relationships between dental attendance patterns, oral health behaviour and the current barriers to dental care. Br. Dent. J. 2013, 214, 25-32. [CrossRef] [PubMed]

6. Corah, N.L. Development of a dental anxiety scale. J. Dent. Res. 1969, 48, 596. [CrossRef]

7. Humphris, G.M.; Morrison, T.; Lindsay, S.J. The Modified Dental Anxiety Scale: Validation and United Kingdom norms. Community Dent. Health 1995, 12, 143-150. [PubMed]

8. Kleinknecht, R.A.; Klepac, R.K.; Alexander, L.D. Origins and characteristics of fear of dentistry. J. Am. Dent. Assoc. 1973, 86, 842-848. [CrossRef] [PubMed]

9. Brand, H.S.; Gortzak, R.A.; Abraham-Inpijn, L. Anxiety and heart rate correlation prior to dental checkup. Int. Dent. J. 1995, 45, 347-351. [PubMed]

10. Caprara, H.J.; Eleazer, P.D.; Barfield, R.D.; Chavers, S. Objective measurement of patient's dental anxiety by galvanic skin reaction. J. Endod. 2003, 29, 493-496. [CrossRef] [PubMed]

11. Gadve, V.R.; Shenoi, R.; Vats, V.; Shrivastava, A. Evaluation of Anxiety, Pain, and Hemodynamic Changes during Surgical Removal of Lower Third Molar under Local Anesthesia. Ann. Maxillofac. Surg. 2018, 8, 247-253. [CrossRef]

12. Wishart, G.C.; Campisi, M.; Boswell, M.; Chapman, D.; Shackleton, V.; Iddles, S.; Hallett, A.; Britton, P.D. The accuracy of digital infrared imaging for breast cancer detection in women undergoing breast biopsy. Eur. J. Surg. Oncol. 2010, 36, 535-540. [CrossRef]

13. Bagavathiappan, S.; Philip, J.; Jayakumar, T.; Raj, B.; Rao, P.N.; Varalakshmi, M.; Mohan, V. Correlation between plantar foot temperature and diabetic neuropathy: A case study by using an infrared thermal imaging technique. J. Diabetes Sci. Technol. 2010, 4, 1386-1392. [CrossRef]

14. Bagavathiappan, S.; Saravanan, T.; Philip, J.; Jayakumar, T.; Raj, B.; Karunanithi, R.; Panicker, T.M.; Korath, M.P.; Jagadeesan, K. Infrared thermal imaging for detection of peripheral vascular disorders. J. Med. Phys. 2009, 34, 43-47. [PubMed]

15. Jones, B.F. A reappraisal of the use of infrared thermal image analysis in medicine. IEEE Trans. Med. Imaging 1998, 17, 1019-1027. [CrossRef] [PubMed]

16. Tattersall, G.J. Infrared thermography: A non-invasive window into thermal physiology. Comp. Biochem. Physiol. A Mol. Integr. Physiol. 2016, 202, 78-98. [CrossRef]

17. Gratt, B.M.; Graff-Radford, S.B.; Shetty, V.; Solberg, W.K.; Sickles, E.A. A 6-year clinical assessment of electronic facial thermography. Dentomaxillofac. Radiol. 1996, 25, 247-255. [CrossRef] [PubMed]

18. Barbosa, J.S.; Amorim, A.; Arruda, M.; Medeiros, G.; Freitas, A.; Vieira, L.; Melo, D.P.; Bento, P.M. Infrared thermography assessment of patients with temporomandibular disorders. Dentomaxillofac. Radiol. 2020, 49, 20190392. [CrossRef] [PubMed] 
19. Carson, J.; Rider, T.; Nash, D. A thermographic study of heat distribution during ultra-speed cavity preparation. J. Dent. Res. 1979, 58, 1681-1684. [CrossRef] [PubMed]

20. Facco, E.; Gumirato, E.; Humphris, G.; Stellini, E.; Bacci, C.; Sivolella, S.; Cavallin, F.; Zanette, G. Modified Dental Anxiety Scale: Validation of the Italian version. Minerva Stomatol. 2015, 64, 295-307.

21. Lahti, S.; Tuutti, H.; Hausen, H.; Kääriäinen, R. Comparison of ideal and actual behavior of patients and dentists during dental treatment. Community Dent. Oral. Epidemiol. 1995, 23, 374-378. [CrossRef] [PubMed]

22. De Jongh, A.; Muris, P.; ter Horst, G.; Van Zuuren, F.J.; De Wit, C.A. Cognitive correlates of dental anxiety. J. Dent. Res. 1994, 73, 561-566. [CrossRef] [PubMed]

23. Scandurra, C.; Gasparro, R.; Dolce, P.; Bochicchio, V.; Muzii, B.; Spagnuolo, G.; Marenzi, G.; Sammartino, G.; Maldonato, N.M The Italian validation of the level of exposure-dental experiences questionnaire. Appl. Sci. 2020, 10, 1143. [CrossRef]

24. de Jongh, A.; Fransen, J.; Oosterink-Wubbe, F.; Aartman, I. Psychological trauma exposure and trauma symptoms among individuals with high and low levels of dental anxiety. Eur. J. Oral. Sci. 2006, 114, 286-292. [CrossRef] [PubMed]

25. Oosterink, F.M.; de Jongh, A.; Aartman, I.H. Negative events and their potential risk of precipitating pathological forms of dental anxiety. J. Anxiety Disord. 2009, 23, 451-457. [CrossRef] [PubMed]

26. Armfield, J.M. Cognitive vulnerability: A model of the etiology of fear. Clin. Psychol. Rev. 2006, 26, 746-768. [CrossRef] [PubMed]

27. Carrillo-Diaz, M.; Crego, A.; Armfield, J.M.; Romero-Maroto, M. Assessing the relative efficacy of cognitive and non-cognitive factors as predictors of dental anxiety. Eur. J. Oral. Sci. 2012, 120, 82-88. [CrossRef] [PubMed]

28. Scandurra, C.; Gasparro, R.; Dolce, P.; Bochicchio, V.; Muzii, B.; Sammartino, G.; Marenzi, G.; Maldonato, N.M. The role of cognitive and non-cognitive factors in dental anxiety: A mediation model. Eur. J. Oral. Sci. 2021, e12793. [CrossRef]

29. Kozlowska, K.; Walker, P.; McLean, L.; Carrive, P. Fear and the Defense Cascade: Clinical Implications and Management. Harv. Rev. Psychiatry 2015, 23, 263-287. [CrossRef] [PubMed]

30. Goldstein, D.S. Adrenal responses to stress. Cell. Mol. Neurobiol. 2010, 30, 1433-1440. [CrossRef] [PubMed]

31. Steimer, T. The biology of fear- and anxiety-related behaviors. Dialogues Clin Neurosci. 2002, 4, 231-249. [PubMed]

32. Ulrich-Lai, Y.M.; Herman, J.P. Neural regulation of endocrine and autonomic stress responses. Nat. Rev. Neurosci. 2009, 10, 397-409. [CrossRef]

33. Ring, E.F.; Ammer, K. Infrared thermal imaging in medicine. Physiol. Meas. 2012, 33, R33-R46. [CrossRef]

34. Lahiri, B.B.; Bagavathiappan, S.; Jayakumar, T.; Philip, J. Medical applications of infrared thermography: A review. Infrared Phys. Technol. 2012, 55, 221-235. [CrossRef] [PubMed]

35. Salazar-López, E.; Domínguez, E.; Juárez Ramos, V.; de la Fuente, J.; Meins, A.; Iborra, O.; Gálvez, G.; Rodríguez-Artacho, M.A.; Gómez-Milán, E. The mental and subjective skin: Emotion, empathy, feelings and thermography. Conscious. Cogn. 2015, 34, 149-162. [CrossRef]

36. Genno, H.; Ishikawa, K.; Kanbara, O.; Kikumoto, M.; Fujiwara, Y.; Suzuki, R.; Osumi, M. Using facial skin temperature to objectively evaluate sensations. Int. J. Ind. Ergon. 1997, 19, 161-171. [CrossRef]

37. Matsuura, H. Systemic complications and their management during dental treatment. Int. Dent. J. 1989, 39, $113-121$.

38. Yuan, S.; Freeman, R.; Hill, K.; Newton, T.; Humphris, G. Communication, Trust and Dental Anxiety: A Person-Centred Approach for Dental Attendance Behaviours. Dent. J. 2020, 8, 118. [CrossRef] [PubMed]

39. Appukuttan, D.P. Strategies to manage patients with dental anxiety and dental phobia: Literature review. Clin. Cosmet. Investig. Dent. 2016, 8, 35-50. [CrossRef] [PubMed]

40. Lin, C.S.; Wu, S.Y.; Yi, C.A. Association between Anxiety and Pain in Dental Treatment: A Systematic Review and Meta-analysis. J. Dent. Res. 2017, 96, 153-162. [CrossRef] [PubMed]

41. Xu, J.L.; Xia, R. Influence factors of dental anxiety in patients with impacted third molar extractions and its correlation with postoperative pain: A prospective study. Med. Oral. Patol. Oral. Cir. Bucal. 2020, 25, e714-e719. [CrossRef] [PubMed] 